\title{
The role of BRCA1 in non-small cell lung cancer
}

\author{
Mariam Gachechiladze, Josef Skarda
}

\begin{abstract}
Background. BRCA1 (Breast cancer 1) was previously identified as a breast and ovarian cancer susceptibility gene, but recently gained a major scientific interest as a prognostic and/or predictive marker for various tumors, including non-small cell lung cancer (NSCLC), which is the leading cause of cancer related mortality in the world. We aimed to review the role of BRCA1 in NSCLC based on currently available literature.

Methods. We performed the literature search in Pubmed database, using key words: BRCA1, non small cell lung cancer, chemotherapy, drug resistance. Articles published in English were selected for review.

Results. Research papers are mainly focused on BRCA1 mRNA expression studies in response to DNA damaging chemotherapy. Several articles about genetic and epigenetic changes of BRCA1 in NSCLC were also available.

Conclusions. BRCA1 is a multifunctional tumor supressor protein, which plays a key role in essential cellular processes and modulates the cellular response to cytotoxic chemotherapy. With the difference from breast and ovarian cancer, BRCA1 has no role in NSCLC cancerogenesis and mainly discussed as a promising genomic marker for customized chemotherapy in NSCLC patients.
\end{abstract}

Key words: BRCA1, non-small cell lung cancer, chemotherapy

Received: September 8, 2011; Accepted after revision: April 13, 2012; Available online: June 1, 2012 http://dx.doi.org/10.5507/bp.2012.049

Department of Clinical and Molecular Pathology, Faculty of Medicine and Dentistry, Palacky University Olomouc, Czech Republic Corresponding author: Mariam Gachechiladze, e-mail: marisa_gachechiladze@yahoo.com

\section{INTRODUCTION}

BRCA1 (Breast cancer 1) used to be identified as a breast and ovarian cancer susceptibility gene but recently it has acquired scientific interest as a prognostic and predictive marker for various tumors, including non-small-cell lung cancer (NSCLC), the leading cause of cancer related mortality in the world, in both men and women ${ }^{1}$. The majority of newly diagnosed NSCLC cases (75-85\%) are at an advanced stage with a median survival time of only $10-11$ months $^{2,3}$ and the five year survival rate is still approximately only $16 \%$ across all stages ${ }^{4}$. An essential step to reducing the mortality from NSCLC is an understanding of the molecular basis of its progression, metastases and drug resistance.

BRCA1 is a multifunctional tumor suppressor protein, which plays a key role in essential cellular processes, such as cell cycle regulation, replication, mitotic spindle assembly, transcription regulation and higher chromatin hierarchical control ${ }^{5}$, as well as DNA damage response (DDR) and apoptosis ${ }^{6}$. Hence, besides its tumor suppression function, BRCA1 also modulates the cellular response to cytotoxic chemotherapy. As drug resistance is a major impediment in the successful treatment of NSCLC, BRCA1 is actively investigated as a predictive marker for NSCLC patients. This review focuses on the possible role of BRCA1 in NSCLC, based on the currently available literature.

\section{Mutations, Epigenetic changes and genetic variations of BRCA1 in NSCLC}

Germline mutations of BRCA1

Germline mutations of BRCA1 are associated with increased risk of breast, ovarian, and more recently, prostate cancer $^{7.9}$ but are not found in lung cancer.

\section{BRCA1 gene methylation}

BRCA1 mutations and epigenetic changes are present in either hereditary or sporadic forms of breast and ovarian tumors. Decreased BRCA1 mRNA and protein expression due to promoter hypermethylation are also found in NSCLC patients. However it is not a frequent event. Lee et al. suggested that promoter hypermethylation is the predominant mechanism for inactivation of the BRCA1 in NSCLC and Marsit et al. suggested that BRCA1 promoter hypermethylation might be specific to some subtypes of NSCLC. The frequency of BRCA1 promoter methylation was found to be only $4 \%(6 / 158)$ in NSCLC, with $5 / 84$ adenocarcinomas and 1/14 large cell carcinomas methylated and no methylation present in lung squamous cell carcinoma $(0 / 60)\left(\right.$ ref. $\left.^{10-11}\right)$. According to Lee at al., $30 \%$ of NSCLC tumors showed promoter hypermethylation in BRCA1 whereas no or low methylation was found in their matched normal lung tissue. A high concordance was observed between alterations in protein and mRNA expression and promoter hypermethylation of the BRCA1 gene. It is worth mentioning that in this study, low BRCA1 
protein expression occurred primarily in patients suffering from adenocarcinoma types of NSCLC $(P=0.014)$, which is somewhat in line with Marsit et al. Some other studies also found BRCA1 promoter hypermethylation in the normal lung tissue of $2.56 \%$ NSCLC patients ${ }^{12}$. This might be one of the early events in lung carcinogenesis but this concept is the subject of future studies.

\section{Germ line variations of BRCA1}

The analysis of single nucleotide polymorphisms (SNPs) and haplotypes in cancer research have pleotropic implications for clinical and public health issues, as well as cancer biology. HT Kim et al. evaluated the associations of four tagging BRCA1 polymorphisms ([S1613G, IVS131893 (A>C), IVS12-1207 (C>T), and IVS12+112 (C>A)]) and their haplotypes (AACC, AACA, GCTC, GATC, and AATC) with treatment outcome in 300 NSCLC patients at stages IIIA (16\%), IIIB (31\%), and IV (53\%). The survival of patients with two copies of the AACC (wild-type) haplotype was significantly shorter than that of patients with zero to one copy (MST, $8.47 v 14.57$ months; logrank $P=0.0066$ ), even after adjustment for body weight loss, performance status, stage, second-line treatment, and radiation therapy (hazard ratio $=2.097 ; 95 \% \mathrm{CI}, 1.339$ to 3.284). Four other haplotypes (AACA, GCTC, GATC, and AATC) were rare and there was no demonstrated association between any of them and survival time (data not shown) (ref. $\left.{ }^{11}\right)$.

\section{BRCA1 as a modulator of chemotherapy response in NSCLC patients}

A number of studies in vitro, mouse model and recently from clinical settings, support the concept that BRCA1 mRNA and protein levels, might be a useful biomarker for chemotherapy response in breast, ovarian and also lung cancer. BRCA1 plays a central role in DNA repair and also in mitotic spindle assembly. Hence a lower expression level is predictive of better survival in treatment with DNA-damaging agents (i.e.platinum), whereas a higher level might indicate benefit from anticancer drugs that act on tubulin, such as taxanes ${ }^{13-16}$.

\section{BRCA1 mRNA expression studies}

The Rosells group was the first to examine the potential predictive value of BRCA1 mRNA expression in resected specimens from 55 stage IIB, IIIA and IIIB NSCLC patients treated with neoadjuvant gemcitabine/ cisplatin followed by surgery. They examined BRCA1 mRNA expression in formalin-fixed paraffin-embedded tissue sections by real-time quantitative polymerase chain reaction (RT-QPCR). BRCA1 was detected in all tumors, although there was a considerable variation in its level of expression, compared with $\beta$-actin as internal ranging control. BRCA1 expression was divided into quartiles. Median survival, was not reached for the 15 patients in the bottom quartile $(0.28$ to 0.61$)$, whereas for the 28 patients in the two middle quartiles ( 0.65 to $1.20,1.23$ to 2.37 ) it was 37.8 months (95\% CI, 10.6-65), and for the 12 patients in the top quartile $(2.45$ to 10.43$)$ it was 12.7 months (95\% CI, 0.28-28.8) (P 5 0.01)). Five patients who attained a complete pathologic response (T0N0) were all in the bottom quartile. Conversely, in the majority of patients with high BRCA1 levels, no clinical or pathologic down staging was found following chemotherapy and surgery. Also, the comparison with pathologic stage, showed that patients in the bottom quartile had a decreased risk of death compared with those in the top quartile (HR 5 0.294; 95\% CI, 0.10-0.83; P 5 0.020), and those in the two middle quartiles also had a decreased risk of death, compared with those in the top quartile. A similar pattern was observed according to clinical stage ${ }^{17}$.

The retrospective analysis of the prognostic value of BRCA1, together with RRM1 and RRM2 mRNA expression in 96 histologically confirmed inoperable stage IIIB and IV NSCLC patients treated with first-line gemcitabine plus docetaxel, showed that the majority of responders had high BRCA1 expression. In contrast to the pattern observed with first-line therapy, low levels of BRCA1 were significantly associated with the lowest risk of progression to second-line therapy ${ }^{18}$. BRCA1 mRNA expression levels were inversely correlated with sensitivity to cisplatin in malignant pleural effusions of NSCLC patients $(P=0.014$, $\mathrm{r}=0.541)$, while patients with higher BRCA1 mRNA expression levels had a higher sensitivity to docetaxel compared with those with lower expression levels (NSCLC: $P=0.008, \mathrm{r}=-0.573)\left(\right.$ ref. $\left.^{19}\right)$.

Two trials confirmed the prognostic relevance of BRCA1 expression. In the first study,

126 chemotherapy-naive patients who had undergone surgical resection had decreased OS associated with increased BRCA1 expression (hazard ratio 1.98; 95\% CI 1.11-6.00; $P=0.02$ ). In patients with low levels of BRCA1, median survival was not attained while it was 29 months (95\% CI, 22.2-35.7 months) for those with high levels $(P=0.04)$ (ref. $\left.{ }^{20}\right)$. The second study included patients with stage IV adenocarcinoma. Patients with epidermal growth factor receptor (EGFR) mutations received erlotinib, whereas patients without EGFR mutations received customized chemotherapy, including cisplatin/ gemcitabine (low BRCA1 levels), cisplatin/docetaxel (intermediate BRCA1 levels), or docetaxel alone (high BRCA1 levels). Median OS was not attained for patients with EGFR mutations and in patients with low levels of BRCA1 and RAP80 (ref. ${ }^{21}$ ). In contrast to these findings, the Bio-FAST study showed inconclusive results for BRCA1 $\left(\right.$ ref. $^{22}$ ). Therefore, the role of BRCA1 as a predictive marker for chemotherapy response in patients with NSCLC is still not clear and needs to be evaluated by further studies. Also, for practical purposes study of BRCA1 protein expression, by immunohistochemistry is more valuable for precise evaluation of individual patient prognosis. 
BRCA1 protein expression studies

Protein expression examined using immunohistochemistry has major diagnostic and practical significance in the study of lung cancers. However, there are few studies, using BRCA1 immunohistochemistry. The first report using immunohistochemical staining of BRCA1 was for a total of 33 patients in a randomized phase III trial comparing cisplatin-gemcitabine and epirubicin-gemcitabine as first-line treatment in advanced NSCLC. Patients were selected according to short ( $<26$ weeks) or a longer (>78 weeks) survival times. Anti-BRCA, clone MS110, 1:25 (Oncogene, Boston, MA, USA) was used for the detection of BRCA1 protein. A biopsy with more than $10 \%$ of cells with positive nuclear or membrane staining was defined as positive. In ninety percent of patients the tumor showed a positive nuclear BRCA1 staining. Tumor response rate was not significantly different in patient biopsies with positively or negatively scored BRCA1. Also, the percentage of tumor cells positively stained for ERCC1, hRad51, and BRCA was similar in responders versus non-responders. Additionally, no differences in percentage of positively stained cells were found between responders and non-responders treated with cisplatingemcitabine or epirubicin-gemcitabine. The percentage of biopsies positive for BRCA1 was similar in patients with a short or long overall survival after chemotherapy. In addition, the percentage of positive cells not correlating with treatment regimen/ was similar in patients with short and long overall survival treated with cisplatin-gemcitabine or epirubicin-gemcitabine ${ }^{23}$. Another study of 98 tumors from NSCLC patients using monoclonal antibodies for BRCA1-8F7 (1:500; GeneTex, San Antonio, TX), showed the same results ${ }^{20}$.

The quantification of expression of $\mathrm{ABC}$ transporter (BCRP, MRP2) proteins and DNA repair proteins (ERCC1, BRCA1) by immunohistochemical staining of tumor biopsy, specimens collected before platinum based chemotherapy, showed no significant associations between BRCA1 expression and response to chemotherapy, or survival. This study used the same clone of BRCA1 antibody (cloneMS110) ( ref. $^{24}$ ).

One study evaluated protein expression profiles by immunohistochemistry on surgical specimens of 82 NSCLC patients who underwent platinum- and taxane-based neoadjuvant chemotherapy. The expression levels of proteins were measured semi-quantitatively and the correlations with tumor response, pathologic cell death rate and survival were evaluated. There was no correlation found in this study either. Unfortunately the BRCA1 clone used was not described, unlike other antibodies used in this study.

On the other hand, changes in BRCA1 mRNA expression might be reflected in changes in protein expression and use of appropriate antibody or panel of antibodies could reveal these changes.

We first described the expression of the phosphorylated form of the BRCA1 protein, in association with NSCLC patient survival. BRCA1 S1524 nuclear positiv- ity was significantly correlated with longer OS and DFS in stage I and II patients $(P<0,001)$ (Fig.1 and 2), while OS and DFS were shorter in S1524 positive stage III and IV patients $(P=0,001)$. From our study results, we concluded that BRCA1 protein study in NSCLC still makes sense but requires more precise antibodies for detecting the functional form of BRCA1.

\section{CONCLUSION}

To the best of our knowledge of the published literature, BRCA1 still remains a potential genomic marker for NSCLC patients. The main weakness of BRCA1 mRNA studies is the small sample size and for protein studies, lack of proper antibody.

\section{ACKNOWLEDGEMENTS}

This publication was supported by a grant MSM 6198959216, IGA MZ CR NS/9959-3, IGA MZ 10285-3 200, NS/10259/3/2009 and project Biomedicine for regional development and human resources (BIOMEDEG) C.1.05/2.1.00/01.0030. Mariam Gachechiladze was also supported by GACR 303/09/HO48 from the Grant Agency of the Czech Republic and LF_2010_006.

\section{CONFLICT OF INTEREST STATEMENT}

Author's conflict of interest disclosure: The authors stated that there are no conflicts of interest regarding the publication of this article.

\section{REFERENCES}

1. Jemal A, Siegel R, Ward E, Hao Y, Xu J, Murray T, Thun MJ. Cancer statistics 2008. CA: A Cancer Journal for Clinicians 2008;58:71-96.

2. Fossella FV, DeVore R, Kerr RN, Crawford J, Natale RR, Dunphy F, Kalman L, Miller V, Lee JS, Moore M, Gandara D, Karp D, Vokes E, Kris M, Kim Y, Gamza F, Hammershaimb L. Randomized, multinational, phase III study of docetaxel plus platinum combinations versus vinorelbine plus cisplatin for advanced non-small-cell lung cancer: the TAX 326 study group. Journal of Clinical Oncology 2003;21:301624.

3. Scagliotti GV, Parikh P, von Pawel J, Biesma B, Vansteenkiste J, Manegold C, Serwatowski P, Gatzemeier U, Digumarti R, Zukin M, Lee JS, Mellemgaard A,Park K, Patil S, Rolski J, Goksel T, de Marinis F, Simms L, Sugarman KP, Gandara D. Phase III study comparing cisplatin plus gemcitabine with cisplatin plus pemetrexed in chemotherapy-naive patients with advanced-stage non-small-cell lung cancer. Journal of Clinical Oncology 2008;26:3543-51.

4. Delbaldo C, Michiels S, Rolland E, Syz N, Soria JC, Le Chevalier T, Pignon JP. Second or third additional chemotherapy drug for nonsmall cell lung cancer in patients with advanced disease. Cochrane Database Syst Rev 2007;4:CD004569.

5. Rosen EM, Fan S, Isaacs C. BRCA1 in hormonal carcinogenesis: basic and clinical research. Endocrine-Related Cancer 2005;12(3):533-48.

6. Yarden RI, Papa MZ. BRCA1 at the crossroad of multiple cellular pathways: approaches for therapeutic interventions. Molecular Cancer Therapeutics 2006;5(6):1396-1404.

7. Kalow W, Tang BK, Endrenyi L. Hypothesis: comparisons of inter- and 
intra-individual variations can substitute for twin studies in drug research. Pharmacogenetics 1998;8:283-9.

8. Parmigiani G, Garrett-Mayer ES, Anbazhagan R, Gabrielson E. A. Crossstudy comparison of gene expression studies for the molecular classification of lung cancer. Clinical Cancer Research 2004;10:29227.

9. Potti A, Mukherjee S, Petersen R, Dressman HK, Bild A, Koontz J, Kratzke R, Watson MA, Kelley M, Ginsburg GS, West M, Harpole DH, Nevins JR. A genomic strategy to refine prognosis in early-stage non-small-cell lung cancer. New England Journal of Medicine 2006;355:570-80.

10. Carmen J, Marsit CJ, Liu M, Nelson HH, Posner M, Suzuki M, and Kelsey KT. Inactivation of the Fanconi anemia/BRCA pathway in lung and oral cancers: implications for treatment and survival. Oncogene 2004;23,1000-4.

11. Lee M, Tseng RC, Hsu HS, Chen JY, Tzao C, Ho WL, Wang YC. Epigenetic Inactivation of the Chromosomal Stability Control Genes BRCA1, BRCA2, and XRCC5 in Non-Small Cell Lung Cancer. Clinical Cancer Research 2007;13:832-8.

12. Wang Y, Zhang D, Zheng W, Luo J, Bai Y, Lu Z. Multiple gene methylation of non small cell lung cancers evaluated with 3-dimensional microarray. Cancer 2008;6:1325-36.

13. Damsma GE, Alt A, Brueckner F, Carell T, Cramer P. Mechanism of transcriptional stalling at cisplatin-damaged DNA. Nature Structura and Molecular Biology 2007;12:1127-33.

14. Basu A, Krishnamurthy S. Cellular Responses to cisplatin-induced DNA damage. Journal of nucleic acids 2010;2010:1-16. doi:10.4061/2010/201367

15. Wang Y, Cortez D, Yazdi P, Neff N, Elledge SJ, Qin J. BASC, a super complex of BRCA1-associated proteins involved in the recognition and repair of aberrant DNA structures. Genes \& Development 2000;14:927-39.

16. Yang ES, Xia F. BRCA1 16 years later: DNA damage-induced BRCA1 shuttling. FEBS Journal 2010;277(15):3079-85.

17. Taron M, Rosell R, Felip E, Mendez P, Souglakos J, Ronco MS, Quralt C, Majo J, Sanchez JM, Sanchez JJ, Maestre J. BRCA1 mRNA expression levels as an indicator of chemoresistance in lung cancer. Human Molecular Genetics2004;13(20):2443-9.

18. Boukovinas I, Papadaki C, Mendez P, Taron M, Mavroudis D, Koutsopoulos A, Sanchez-Ronco M, Sanchez JJ, Trypaki MM,
Staphopoulos E, Georgoulias V, Rosell R, Souglakos J. Tumor BRCA1, RRM1 and RRM2 mRNA Expression Levels and Clinical Response to First-Line Gemcitabine plus Docetaxel in Non-Small-Cell Lung Cancer Patients. PLoS ONE2008;3(11):e3695.

19. Wang L, Wei J, Qian X, Yin H, ZhaoY, Yu L, Wang T, Liu B. ERCC1 and BRCA1 mRNA expression levels in metastatic malignant effusions is associated with chemosensitivity to cisplatin and/or docetaxel. BMC Cancer 2008;8:97.

20. Rosell R, Skrzypski M, Jassem E, Taron M, Bartolucci R, Sanchez JJ, Mendez P, Chaib I, Perez-Roca L, Szymanowska A, Rzyman W, Puma F, Kobierska-Gulida F, Farabi R, Jassem J. BRCA1: a novel prognostic factor in resected nonsmall-cell lung cancer; PLoS One2007;2:e1129.

21. Rosell R, Perez-Roca L, Sanchez JJ, Cobo M, Moran T, Chaib I, Provencio M, Domine M, Sala MA, Jimenez U, Diz P, Barneto I, Macias $J A$, Peñas R, Catot S, Isla D, JM Sanchez, Ibeas R, Lopez-Vivanco G, Oramas J, Mendez P, Reguart N, Blanco R, Taron M. Customized treatment in nonsmallcell lung cancer based on EGFR mutations and BRCA1 mRNA expression. PLoS One2009;4:e5133.

22. Tiseo M, Bordi P, Bortesi B. ERCC 1/BRCA1 protein expressions and ERCC1/ XPD/XRCC1-3 gene polymorphisms as predictor factors of outcome in stage IIIB-IV nonsmall cell lung cancer (NSCLC) patients treated in first-line chemotherapy with or without cisplatin. Journal of Thoracic Oncol 2009;4:D7.5.

23. Wachtersa FM, Wong LSM. ERCC1, hRad51, and BRCA1 protein expression in relation to tumour response and survival of stage III/IV NSCLC patients treated with chemotherapy. Lung Cancer 2005;50:211-9.

24. Shuji Ota, Genichiro Ishii, Immunohistochemical expression of BCRP and ERCC1 in biopsy specimen predicts survival in advanced nonsmall-cell lung cancer treated with cisplatin-based chemotherapy. Lung Cancer 2009;64:98-104.

25. Kanga $\mathrm{CH}$, Jangb BG, Kimc DW, Chungb DH, Kima YT, Jheond $\mathrm{S}$, Sungd CW, Kima JH. The prognostic significance of ERCC1, BRCA1, XRCC1, and $\beta$ III-tubulin expression in patients with non-small cell lung cancer treated by platinum- and taxane-based neoadjuvant chemotherapy and surgical resection. Lung Cancer2010; 68(3):47883. 